\section{Bacterial Selenium Methylation in Sulfur Dominated Environments}

MATHIEU MARTINEZ, YING LIU AND MARKUS LENZ

Institute for Ecopreneurship, School of Life Sciences, University of Applied Sciences and Arts Northwestern Switzerland (FHNW)

Presenting Author: mathieu.martinez@fhnw.ch

Selenium (Se) is an essential micronutrient with a very narrow range between deficiency, essentiality, and toxicity. Its mobility, bioavailability, bioaccumulation, and toxicity depend mainly on its speciation. Therefore, understanding Se speciation is of major importance in predicting its biogeochemical behavior. In the environment, the methylation of Se is performed by a wide range of organisms (bacteria, fungi, algae, plants and animals) and leads to the formation of biogenic volatiles species (e.g. dimethylselenide (DMSe), and dimethyl diselenide (DMDSe)) which represent major selenium fluxes to the atmosphere. However, most studies on Se environmental transformations focused on systems characterized by high Se concentrations which is not necessarily representative of environmental conditions. In soil systems and at sediment-water interfaces, sulfur (S) concentrations often exceed Se concentrations by orders of magnitude. This work investigated the role of S in the control of Se speciation. It is demonstrated that the sulfur status of bacteria crucially determines the amount of Se volatilized through methylation. Indeed, when Pseudomonas tolaasii was supplemented with cystine (but not methionine), a major proportion of the Se $(\sim 48 \%)$ was channeled to thus far unknown, non-volatile Se species at the expense of the previously formed dimethyldiselenide and dimethylselenylsulfide (accounting for less than $4 \%$ of total Se) (Figure 1). We used a combination of mass spectrometry techniques (IC-ICP-MS, SPME-GC-MS and ESI-qTOF-MS) to determine speciation of Se and identify a novel Se metabolite (Figure 2). Our results shed light on sulfur and selenium cycles inter-connections and on novel Se species. The implications of the new Se species on Se cycling in such environments is discussed.



Figure 1. Conversion of selenite to volatile methylated species (A), metabolite 1 (B) and metabolite $2(C)$ in Pseudomonas cultures at stationary phase $(\sim 18 \mathrm{~h})$ supplemented with methionine alone
(Met), cystine alone (Cy), methionine and cystine (Met+Cy) and without supplementation (Control).
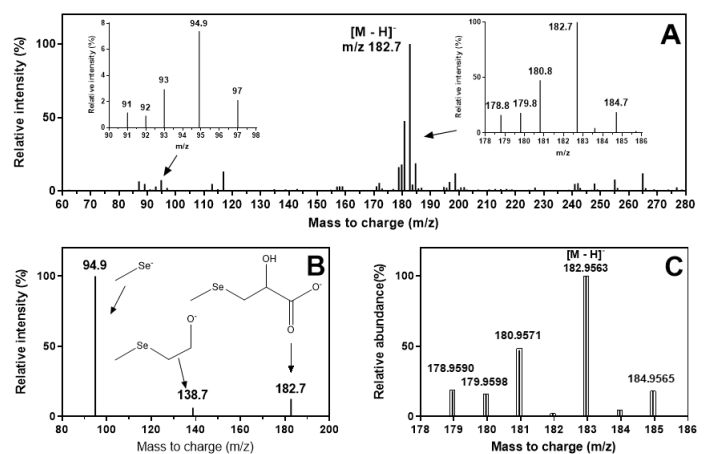

Figure 2. Mass spectrometric analysis of the collected fraction corresponding to Figure 2. Mass spectrometric analysis of the collected fraction corresponding to
unknown1. ESI-ion-trap-MS analysis (A) full mass scan and zoom on selected mass spectra (inserts) demonstrating isotopic pattern of one Se atom ;

(B) ESI-MS $\mathrm{S}^{2}$ ragmentation of $\mathrm{m} / \mathrm{z} 183$; (C) ESI-Q-TOF-MS high resolution mass spectra in negative mode (bars) and calculated mass distribution of $\left[\left(\mathrm{C}_{4} \mathrm{H}_{8} \mathrm{O}_{3} \mathrm{Se}\right)-\mathrm{H}\right]$ (boxes). 